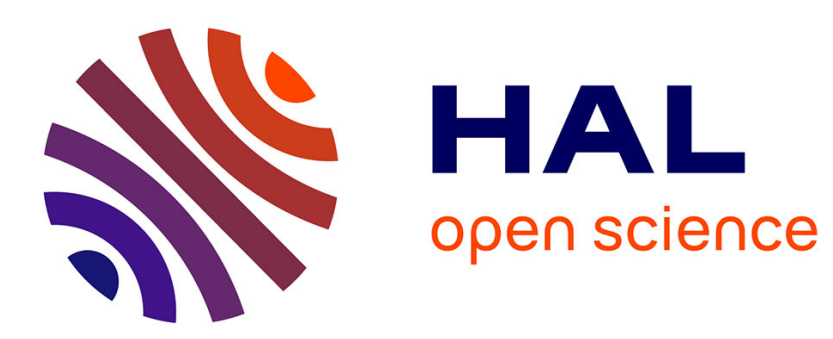

\title{
A partitioned fluid-structure algorithm for elastic thin valves with contact
}

Nuno Diniz dos Santos, Jean-Frédéric Gerbeau, Jean-François Bourgat

\section{To cite this version:}

Nuno Diniz dos Santos, Jean-Frédéric Gerbeau, Jean-François Bourgat. A partitioned fluid-structure algorithm for elastic thin valves with contact. [Research Report] RR-6022, INRIA. 2006, pp.23. inria00111991v2

\section{HAL Id: inria-00111991 https://hal.inria.fr/inria-00111991v2}

Submitted on 21 Nov 2006

HAL is a multi-disciplinary open access archive for the deposit and dissemination of scientific research documents, whether they are published or not. The documents may come from teaching and research institutions in France or abroad, or from public or private research centers.
L'archive ouverte pluridisciplinaire HAL, est destinée au dépôt et à la diffusion de documents scientifiques de niveau recherche, publiés ou non, émanant des établissements d'enseignement et de recherche français ou étrangers, des laboratoires publics ou privés. 
INSTITUT NATIONAL DE RECHERCHE EN INFORMATIQUE ET EN AUTOMATIQUE

\section{A partitioned fluid-structure algorithm for elastic thin valves with contact}

Nuno Diniz dos Santos — Jean-Frédéric Gerbeau — Jean-François Bourgat

$$
\mathbf{N}^{\circ} 6022
$$

Novembre 2006

Thème $\mathrm{BIO}$

\section{apport}

de recherche 



\title{
A partitioned fluid-structure algorithm for elastic thin valves with contact
}

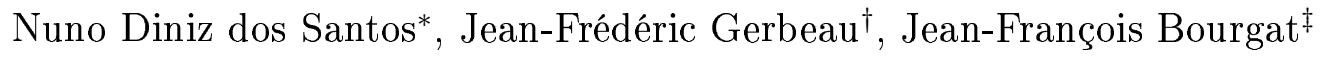 \\ Thème BIO - Systèmes biologiques \\ Projets REO \& BANG \\ Rapport de recherche n 6022 - Novembre 2006 - 23 pages
}

\begin{abstract}
We present a numerical method to simulate the movements of a thin valve immersed in an incompressible viscous fluid. The fluid and structure meshes are not matching: the kinematic continuity is imposed using Lagrange multipliers. The method therefore belongs to the "Fictitious Domain" (FD) family. This approach allows very large displacements. We propose a partitioned fluid-structure algorithm which keeps the fluid and structure solvers independent and which is able to manage contact without assuming that the structure solvers include contact capabilities. Various numerical tests are proposed. In particular, when the displacement is moderate, we show that the results are in good agreement with Arbitrary Lagrangian Eulerian (ALE) simulations. We then present results with contact on a rigid wall. Finally we show that the proposed approach can be mixed with ALE when the wall is elastic.
\end{abstract}

Key-words: fictitious domains, ALE, valves, contact

(MSC:65M60,74K25,76D05,76Z05)

Paper submitted to Computer Methods in Applied Mechanics and Engineering

* INRIA \& Paris VI, Projet REO, email: nuno-miguel.diniz-dos-santos@inria.fr

$\dagger$ INRIA \& Paris VI, Projet REO, email: jean-frederic.gerbeau@inria.fr

$\ddagger$ INRIA Projet BANG, email: jean-francois.bourgat@inria.fr

\author{
Unité de recherche INRIA Rocquencourt \\ Domaine de Voluceau, Rocquencourt, BP 105, 78153 Le Chesnay Cedex (France) \\ Téléphone : +33139635511 -Télécopie : +33139635330
}




\section{Un algorithme fluide-structure partitionné pour des valves elastiques minces avec contact}

Résumé : On présente une méthode numérique pour simuler les mouvements d'une valve mince immergée dans un fluide visqueux incompressible. Les maillages du fluide et de la structure sont indépendants : la continuité cinématique est imposée en utilisant des multiplicateurs de Lagrange. La méthode est donc du type "domaines fictifs" (FD). Cette approche autorise de très grands déplacements de structure. On propose un algorithme de couplage partitionné qui garde les codes fluide et structure indépendants et qui peut traiter des situations de contact sans supposer que cela soit initialement prevu dans le code structure. On présente divers essais numériques. En particulier, quand le déplacement est modéré, on montre que les résultats obtenus avec la méthode de domaines fictifs sont en bon accord avec des simulations basées sur une formulation Arbitraire Lagrange Euler (ALE). On présente également des simulations avec contact de la valve sur une paroi rigide. Enfin on montre que l'approche proposée peut facilement se coupler avec une formulation ALE quand la paroi est élastique.

Mots-clés : domaines fictifs, ALE, valves, contact (MSC:65M60,74K25,76D05,76Z05) 


\section{INTRODUCTION}

This work is motivated by the modelling of biological valves, such as the aortic valve. The models presented here are clearly too simple to handle the complexity of real biological valves. Nevertheless it can be viewed as a first step towards more realistic simulations.

Various approaches have been investigated to model fluid-structure interaction problems involving valves. The Arbitrary Lagrangian Eulerian (ALE) formulation consists in using moving meshes which follow the valve movements. It has been used for example by Jianhai et al. [14 to study the 2D behavior of an artificial valve (rigid body), and by Le Tallec and Mouro [15] to study valves in hydraulic shock absorbers used in cars. In presence of very large displacements, ALE algorithms need frequent remeshing, which may introduce diffusion in the numerical solution. Moreover, by construction, ALE is not adapted to topological changes which occur for example when the valves close.

Other methods are based on a priori fixed meshes. This is the case of the immersed boundary method by Peskin (see for example [19] among many references on the subject). In this approach, fluid and structure are solved simultaneously: the interaction with the structure is taken into account using an external force acting on the fluid. Another possibility is to consider independent meshes for the fluid and the structure. The coupling is then obtained by enforcing the kinematic condition with Lagrange mutlipliers. This is the basic idea of the so-called Fictitious Domain (FD) method which has been much investigated by Glowinski and co-workers (see for example [13, 11, 12]). In fluid-structure interaction problems, the FD method was originally used for rigid particles. But it has also been applied for flexible structures, either using Lagrange multiplier located on the structure surface (see the works by Baaijens and co-workers [1, 4, 17]) or Lagrange multiplier located on the structure volume [21].

If FD has been originally designed for fixed meshes, it appears that it may also be interesting to use it on moving meshes, mixing ALE and FD formulations (see [3]). Indeed, if FD is a powerful method for valves, ALE formulation is more accurate and robust as far as the wall is concerned. In blood flow applications, it is useful to take into account both wall and valve movements: for example, it is shown in [20] that the compliance of the aortic root contributes to the leaflet opening and to the ability of the aortic valve to increase its effective orifice area when necessary.

The present work is mainly based on a formulation similar to the Baaijens formulation [1]. It differs nevertheless on various points. From the mechanical viewpoint, we consider here a genuine thin structure model (Section 2), which is required for surface Lagrange multipliers. Futhermore, we do not neglect the structure inertia. From the numerical viewpoint, we propose a partitioned algorithm instead of a monolithic formulation (Section 2.3) and we propose to handle contacts with an Uzawa algorithm which is well-adapted to the partioned fluid-structure strategy (Section 4.3).

In Section 5, we present various numerical simulations. In particular we propose a quantitative comparison between the ALE and the FD methods for small displacements. We next present simulations involving contact with a rigid straight wall and a test case mixing ALE and FD. 


\section{MODELLING}

\subsection{Fluid equations}

Let $\Omega$ be a $2 \mathrm{D}$ domain. For the moment, $\Omega$ is assumed to be fixed (in Section 5.4 we will show numerical simulations with a time dependent $\Omega$ ). We consider the flow of an homogeneous, viscous and incompressible fluid in $\Omega$ around an elastic valve located on a 1D manifold $\Sigma(t)$. The fluid domain is defined as $\Omega_{F}(t)=\Omega \backslash \Sigma(t)$. The governing equations are the standard incompressible Navier-Stokes equations:

$$
\left\{\begin{aligned}
\rho\left(\frac{\partial \boldsymbol{u}}{\partial t}+\boldsymbol{u} \cdot \nabla \boldsymbol{u}\right)-\eta \Delta \boldsymbol{u}+\nabla p & =0 & & \text { for } x \in \Omega_{F}(t), \\
\operatorname{div} \boldsymbol{u} & =0 & & \text { for } x \in \Omega_{F}(t) .
\end{aligned}\right.
$$

where $\boldsymbol{u}$ is the fluid velocity, $p$ the pressure, $\rho$ the fluid density and $\eta$ the dynamic viscosity. The Cauchy stress tensor is denoted by

$$
\boldsymbol{\sigma}=-p \mathbf{I}+2 \eta \mathbf{D}(\boldsymbol{u}),
$$

where $\mathbf{I}$ is the identity tensor and $\mathbf{D}(\boldsymbol{u})$ is the strain rate $\left(\nabla \boldsymbol{u}+\nabla \boldsymbol{u}^{T}\right) / 2$. We define $\boldsymbol{n}$ as the outward normal on $\partial \Omega_{F}(t)$. For example we may impose the total stress at the inlet $\Gamma_{\text {in }}$

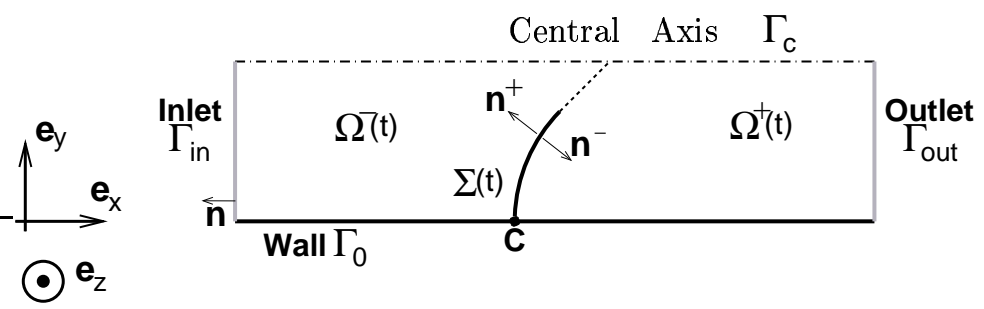

Figure 1: Sketch of the geometry.

and the outlet $\Gamma_{\text {out }}$ :

$$
\left\{\begin{aligned}
\boldsymbol{\sigma} \cdot \boldsymbol{n}(\cdot, t) & =-p_{i n}(\cdot, t) \boldsymbol{n} & & \text { on } & \Gamma_{\text {in }} \times(0, T), \\
\boldsymbol{\sigma} \cdot \boldsymbol{n} & =0 & & \text { on } & \Gamma_{\text {out }} \times(0, T),
\end{aligned}\right.
$$

where $p_{\text {in }}$ is a given function. An alternative choice to represent the injection of fluid would be to impose a velocity profile over $\Gamma_{\text {in }}$ or a flux (see e.g. [9] or [6]). No-slip boundary conditions are imposed on the wall $\Gamma_{0}$ and symmetry boundary conditions on the central axis $\Gamma_{c}$ (see Fig. 1). The system is completed with suitable initial conditions.

The valve $\Sigma(t)$ is assumed to define a natural partition of $\Omega_{F}(t)$ into two subdomains $\Omega^{-}(t)$ and $\Omega^{+}(t)$ (see Fig. 1. On $\Sigma(t)$, we define $\boldsymbol{n}^{+}$(resp. $\boldsymbol{n}^{-}$) as the outgoing normal on $\partial \Omega^{+}$(resp. $\left.\partial \Omega^{-}\right)$, and:

$$
\boldsymbol{f}_{\Sigma}=-\left(\boldsymbol{\sigma}^{+} \cdot \boldsymbol{n}^{+}+\boldsymbol{\sigma}^{-} \cdot \boldsymbol{n}^{-}\right)
$$


where $\boldsymbol{\sigma}^{+}(\mathbf{x})\left(\right.$ resp. $\left.\boldsymbol{\sigma}^{-}(\mathbf{x})\right)$, for $\mathbf{x} \in \Sigma(t)$, is the limit of $\boldsymbol{\sigma}\left(\mathbf{x}-\varepsilon \boldsymbol{n}^{+}\right)$as $\varepsilon$ goes to $0^{+}$(resp. $\left.0^{-}\right)$.

\subsection{Structure equations}

The structure is assumed to be monodimensional and inextensible. Its deformation energy is defined by

$$
W(\boldsymbol{x})=\frac{1}{2} \int_{0}^{L} E I\left|\frac{\partial^{2} \boldsymbol{x}}{\partial s^{2}}\right|^{2} d s
$$

where $E$ denotes the Young Modulus, $I$ the inertia, $\boldsymbol{x}(s)$ is the position vector of a point on the structure's axis. The quantity EI represents the flexural stiffness. The solution is searched in

$$
\begin{aligned}
& K=\left\{\boldsymbol{x} \in\left(C^{1}(0, L)\right)^{2} ; \frac{\partial \boldsymbol{x}}{\partial s} \in\left(L^{2}(0, L)\right)^{2} ;\left|\frac{\partial \boldsymbol{x}}{\partial s}\right|^{2}=1 ;\right. \\
& \left.\boldsymbol{x}(0)=0 \text { and } \frac{\partial \boldsymbol{x}}{\partial s}(0)=0\right\}
\end{aligned}
$$

which is the set of the allowed structure configurations that satisfy the boundary conditions as well as the inextensibility constraint.

Thus, the configurations of the immersed structure are governed by the following problem:

$$
\left\{\begin{array}{l}
\text { Find } \boldsymbol{x}(s, t) \in K \text { such that for all } t \\
\int_{0}^{L} m \frac{\partial^{2} \boldsymbol{x}}{\partial t^{2}} \cdot \boldsymbol{\xi} d s+\int_{0}^{L} E I \frac{\partial^{2} \boldsymbol{x}}{\partial s^{2}} \cdot \frac{\partial^{2} \boldsymbol{\xi}}{\partial s^{2}} d s=\int_{0}^{L} \boldsymbol{f} \cdot \boldsymbol{\xi} d s, \quad \forall \boldsymbol{\xi} \in K,
\end{array}\right.
$$

where $m$ denotes the linear mass of the structure and $f$ the external forces. In the sequel, $f$ will typically be the hydrodynamic force $f_{\Sigma}$. Nevertheless, in Section 4.3, $f$ will also take into account contact forces $\boldsymbol{f}_{c}$.

\subsection{Fluid-structure coupling}

In the configuration at hand, the structure being immersed and having one dimension less than the fluid, the fluid-structure interface coincides with the structure domain $\Sigma(t)$. The fluid-structure coupling is enforced throught the following relations:

$$
\begin{gathered}
\frac{\partial \boldsymbol{x}}{\partial t}(s, t)=\boldsymbol{u}(\boldsymbol{x}(s, t), t), \\
\boldsymbol{f}(s, t)=\boldsymbol{f}_{\Sigma}(\boldsymbol{x}(s, t), t),
\end{gathered}
$$

$f_{\Sigma}$ being defined in (2).

It will be convenient to denote the structure velocity in $\boldsymbol{x}=\boldsymbol{x}(s, t) \in \Sigma(t)$ by

$$
\boldsymbol{u}_{\Sigma}(\boldsymbol{x}, t) \stackrel{\text { def }}{=} \frac{\partial \boldsymbol{x}}{\partial t}(s, t)
$$




\section{DISCRETIZATION}

\subsection{Fluid problem}

\subsubsection{Time discretization of the augmented formulation}

Problem (1) is discretized in time by a semi-implicit Euler scheme:

$$
\left\{\begin{aligned}
\rho \frac{\boldsymbol{u}^{n+1}-\boldsymbol{u}^{n}}{\delta t}+\rho \boldsymbol{u}^{n} \cdot \nabla \boldsymbol{u}^{n+1}+\nabla p^{n+1}-\operatorname{div}\left(2 \eta \mathbf{D}\left(\boldsymbol{u}^{n+1}\right)\right) & =0 \\
\operatorname{div} \boldsymbol{u}^{n+1} & =0 .
\end{aligned}\right.
$$

For the time being, the structure position and velocity are assumed to be known at time $t^{n+1}$ : its configuration is denoted by $\Sigma^{n+1}$, and its velocity by $\boldsymbol{u}_{\Sigma^{n+1}}^{n+1}$. To give a variational formulation of (8), we introduce the following functional spaces:

$$
\begin{aligned}
X & =\left\{\boldsymbol{v} \in\left(H^{1}(\Omega)\right)^{2}, \boldsymbol{v}=0 \text { on } \Gamma_{0}, \boldsymbol{v} \cdot \boldsymbol{n}=0 \text { on } \Gamma_{c}\right\} \\
V & =\left\{\boldsymbol{v} \in X, \operatorname{Tr}_{\Sigma^{n+1}}(\boldsymbol{v})=0, \operatorname{div} \boldsymbol{v}=0\right\} \\
V\left(\boldsymbol{u}_{\Sigma^{n+1}}\right) & =\left\{\boldsymbol{v} \in X, \operatorname{Tr}_{\Sigma^{n+1}}(\boldsymbol{v})=\boldsymbol{u}_{\Sigma^{n+1}}, \operatorname{div} \boldsymbol{v}=0\right\}
\end{aligned}
$$

where $\operatorname{Tr}_{\Sigma^{n+1}}:\left(H^{1}(\Omega)\right)^{2} \longrightarrow\left(H^{1 / 2}\left(\Sigma^{n+1}\right)\right)^{2}$ denotes the trace operator on $\Sigma^{n+1}$.

A first variational formulation of the fluid problem is: find $\boldsymbol{u}^{n+1} \in V\left(\boldsymbol{u}_{\Sigma^{n+1}}^{n+1}\right)$ such that, for all $v \in V$,

$$
\int_{\Omega} \rho \frac{\boldsymbol{u}^{n+1}-\boldsymbol{u}^{n}}{\delta t} \cdot \boldsymbol{v}+\int_{\Omega} \rho \boldsymbol{u}^{n} \cdot \nabla \boldsymbol{u}^{n+1} \cdot \boldsymbol{v}+\int_{\Omega} 2 \eta \mathbf{D}\left(\boldsymbol{u}^{n+1}\right): \mathbf{D}(\boldsymbol{v})=-\int_{\Gamma_{\text {in }}} p_{i n} \boldsymbol{n} \cdot \boldsymbol{v} .
$$

From the discretization viewpoint, it is much more convenient to work with space $X$. Thus, following [11], we introduce the Lagrange multiplier spaces

$$
\begin{aligned}
M & =L^{2}(\Omega), \\
\mathcal{L}^{n+1} & =\left(H^{-1 / 2}\left(\Sigma^{n+1}\right)\right)^{2},
\end{aligned}
$$

corresponding to the two constraints which define the space $V\left(\boldsymbol{u}_{\Sigma^{n+1}}^{n+1}\right)$. We then consider the following variational formulation: find $\left(\boldsymbol{u}^{n+1}, p^{n+1}, \boldsymbol{\lambda}^{n+1}\right) \in X \times M \times \mathcal{L}^{n+1}$ such that, for all $(\boldsymbol{v}, q, \boldsymbol{\mu}) \in X \times M \times \mathcal{L}^{n+1}$,

$$
\begin{aligned}
\int_{\Omega} \rho \frac{\boldsymbol{u}^{n+1}-\boldsymbol{u}^{n}}{\delta t} \cdot \boldsymbol{v}+\int_{\Omega} \rho \boldsymbol{u}^{n} \cdot \nabla \boldsymbol{u}^{n+1} \cdot \boldsymbol{v} & +\int_{\Omega} 2 \eta \mathbf{D}\left(\boldsymbol{u}^{n+1}\right): \mathbf{D}(\boldsymbol{v}) \\
-\int_{\Omega} p^{n+1} \operatorname{div} \boldsymbol{v}+\left\langle\boldsymbol{\lambda}^{n+1}, \operatorname{Tr}_{\Sigma^{n+1}}(\boldsymbol{v})\right\rangle & =-\int_{\Gamma_{i n}} p_{i n} \boldsymbol{n} \cdot \boldsymbol{v} \\
\int_{\Omega} q \operatorname{div} \boldsymbol{u} & =0 \\
\left\langle\boldsymbol{\mu}, \operatorname{Tr}_{\Sigma^{n+1}}\left(\boldsymbol{u}^{n+1}\right)\right\rangle & =\left\langle\boldsymbol{\mu}, \boldsymbol{u}_{\Sigma^{n+1}}^{n+1}\right\rangle
\end{aligned}
$$


where $\langle\cdot, \cdot\rangle$ denotes the duality pairing on $\left(H^{-1 / 2}\left(\Sigma^{n+1}\right)\right)^{2} \times\left(H^{1 / 2}\left(\Sigma^{n+1}\right)\right)^{2}$.

The domain $\Omega$ is subdivided in a left-hand side and a right-hand side, respectively $\Omega^{-}$ and $\Omega^{+}$as indicated on Figure 1 Applying Green's formula in $\Omega^{-}$and $\Omega^{+}$and, using the fact that $\left(\boldsymbol{u}^{n+1}, p^{n+1}\right)$ solves problem (8) almost everywhere in $\Omega^{-}$and $\Omega^{+}$, we obtain the meaning of $\lambda^{n+1}$ :

$$
\lambda^{n+1}=f_{\Sigma}^{n+1},
$$

where $f_{\Sigma}$ is defined in (2). In other words, the Lagrange multiplier $\lambda^{n+1}$ corresponding to the constraint $\boldsymbol{u}^{n+1}=\boldsymbol{u}_{\Sigma^{n+1}}^{n+1}$ represents the jump of the hydrodynamic stress through the valve.

\subsubsection{Space discretization}

The spaces $X$ and $M$ are approximated by the finite element spaces $X_{h}$ and $M_{h}$. The simulations presented in this paper have been performed with $\mathbb{P}_{1} / \mathbb{P}_{1}$ stabilized finite elements. We denote by $N_{F}$ the number of degrees of freedom for each component of the velocity in $X_{h}$.

The 1D structure is defined by $N_{\Sigma}$ discretization points $\left(\mathbf{x}_{i}^{n+1}\right)_{i=1, \ldots, N_{\Sigma}}$ on $\Sigma^{n+1}$. The Lagrange multipliers space $\mathcal{L}^{n+1}$ can be approximated by different ways. For this work, we choose:

$$
\mathcal{L}_{h}^{n+1}=\left\{\boldsymbol{\mu}_{h} \text { measure on } \Sigma^{n+1}, \boldsymbol{\mu}_{h}=\sum_{i=1}^{N_{\Sigma}} \boldsymbol{\mu}_{i} \delta\left(\mathbf{x}_{i}^{n+1}\right), \boldsymbol{\mu}_{i} \in \mathbb{R}^{2}\right\},
$$

where $\delta\left(\mathbf{x}_{i}^{n+1}\right)$ denotes the Dirac measure at point $\mathbf{x}_{i}^{n+1}$. We have, for $\boldsymbol{\mu}_{h} \in \mathcal{L}_{h}$ and $\boldsymbol{v}_{h} \in X_{h}$,

$$
<\boldsymbol{\mu}_{h}, \operatorname{Tr}_{\Sigma^{n+1}}\left(\boldsymbol{v}_{h}\right)>=\sum_{i=1}^{N_{\Sigma}} \boldsymbol{\mu}_{i} \boldsymbol{v}_{h}\left(\mathbf{x}_{i}\right) .
$$

Note that this approach is meaningful after discretization as soon as $X_{h} \subset\left(\mathcal{C}^{0}(\Omega)\right)^{2}$. This is the case with the finite element used in this work, thus (15) is well-defined.

Remark 1 Notice that we use a continuous pressure finite element. A pressure jump being expected through the valve, the use of a discontinuous pressure space may be preferable, as mentioned in [1]. Nevertheless, the valve mesh being independent from the fluid mesh, the pressure jump can a priori occur within an element and this discontinuity cannot be catched, even with a discontinuous pressure finite element (whose discontinuity occurs only at the fluid element boundary). An enrichment of the pressure functions depending on the valve position would be probably more accurate. We do not investigate this issue here. With a sufficiently fine grid, the results we obtained computing the stress jump in a variational way, as explained in Section 4.2, were satisfactory. But we believe that there is room for improvement in the pressure discretization. 


\subsection{Structure problem}

\subsubsection{Time discretization and solution strategy}

The structure is discretized in time with the second order Houbolt scheme [16]. The solution $\boldsymbol{x}^{n}$ is found solving the following problem:

$$
\left\{\begin{array}{c}
\text { Find } \boldsymbol{x}^{n} \in K \text { such that } \\
\int_{0}^{L} m \frac{2 \boldsymbol{x}^{n}-5 \boldsymbol{x}^{n-1}+4 \boldsymbol{x}^{n-2}-\boldsymbol{x}^{n-3}}{\delta t^{2}} \cdot \boldsymbol{\xi} d s+\int_{0}^{L} E I \frac{\partial^{2} \boldsymbol{x}^{n}}{\partial s^{2}} \cdot \frac{\partial^{2} \boldsymbol{\xi}}{\partial s^{2}} d s= \\
\int_{0}^{L} \boldsymbol{f}^{n} \cdot \boldsymbol{\xi} d s
\end{array}\right.
$$

for all $\boldsymbol{\xi} \in K$. The first two steps are initialized with a Crank-Nicolson scheme.

The numerical solution of (16) is equivalent to finding the local minimum, on $K$, of the following functional

$$
\begin{aligned}
J(\boldsymbol{x})= & \frac{1}{\delta t^{2}} \int_{0}^{L} m|\boldsymbol{x}|^{2} d s+\frac{1}{2} \int_{0}^{L} E I\left|\frac{\partial^{2} \boldsymbol{x}}{\partial s^{2}}\right|^{2} d s-\int_{0}^{L} \boldsymbol{f}^{n} \cdot \boldsymbol{x} d s \\
& -\frac{1}{\delta t^{2}} \int_{0}^{L} m\left(-5 \boldsymbol{x}^{n-1}+4 \boldsymbol{x}^{n-2}-\boldsymbol{x}^{n-3}\right) \cdot \boldsymbol{x} d s .
\end{aligned}
$$

To account for the inextensibility constraint $\left|\partial_{s} x\right|=1$, an augmented Lagrangian method is used. Following [2], we introduce the additional variable

$$
\mathbf{q}=\frac{\partial \boldsymbol{x}}{\partial s}, \quad \mathbf{q} \in\left(L^{2}(0, L)\right)^{2}, \quad|\mathbf{q}|^{2}=1 .
$$

We are then brought to the resolution of an equivalent problem, the search of a saddle-point $\left\{\boldsymbol{x}^{n}, \mathbf{q}^{n}, \lambda^{n}\right\}$ of the augmented Lagrangian

$$
\mathscr{L}(\boldsymbol{x}, \mathbf{q}, \lambda)=J(\boldsymbol{x})+\int_{0}^{L} \lambda\left(\frac{\partial \boldsymbol{x}}{\partial s}-\mathbf{q}\right) d s+\frac{r}{2} \int_{0}^{L}\left|\frac{\partial \boldsymbol{x}}{\partial s}-\mathbf{q}\right|^{2} d s .
$$

The resolution is done with an Uzawa algorithm:

$$
\begin{aligned}
& \text { Let } \lambda_{1}^{n}=\lambda^{n-1} \text { and } \boldsymbol{x}_{0}^{n}=\boldsymbol{x}^{n-1}, \text { compute } \\
& \left\{\begin{array}{l}
\mathbf{q}_{p}^{n} \text { which minimizes } \mathscr{L}\left(\boldsymbol{x}_{p-1}^{n}, \mathbf{q}, \lambda_{p}^{n}\right), \text { with }|\mathbf{q}|=1, \\
\boldsymbol{x}_{p}^{n} \text { which minimizes } \mathscr{L}\left(\boldsymbol{x}, \mathbf{q}_{p}^{n}, \lambda_{p}^{n}\right), \\
\lambda_{p+1}^{n}=\lambda_{p}^{n}+r\left(\frac{\partial \boldsymbol{x}_{p}^{n}}{\partial s}-\mathbf{q}_{p}^{n}\right)
\end{array}\right.
\end{aligned}
$$

where $r$ is a fixed parameter. This algorithm converges when $p \rightarrow \infty$ to the saddle-point $\left(\boldsymbol{x}^{n}, \mathbf{q}^{n}, \lambda^{n}\right)$, which means that $\left(\boldsymbol{x}^{n}, \mathbf{q}^{n}\right)$ minimizes $\mathscr{L}\left(\boldsymbol{x}, \mathbf{q}, \lambda^{n}\right)$ and $\lambda^{n}$ maximizes $\mathscr{L}\left(\boldsymbol{x}^{n}, \mathbf{q}^{n}, \lambda\right)$.

In order to find the solution for problem (19) 1 , we solve

$$
\frac{\partial \mathscr{L}}{\partial \mathbf{q}}\left(\boldsymbol{x}_{p-1}^{n}, \mathbf{q}, \lambda_{p}^{n}\right)=0,
$$


noticing that, $J(\boldsymbol{x})$ being independent of $\mathbf{q}$, this solution is obtained by minimizing at each point $s$,

$$
-\left(r \frac{\partial \boldsymbol{x}_{p-1}^{n}}{\partial s}+\lambda_{p}^{n}\right) \cdot \mathbf{q}
$$

over the circle $|\mathbf{q}|=1$. This gives

$$
\mathbf{q}_{n}=\frac{r \frac{\partial \boldsymbol{x}_{p-1}^{n}}{\partial s}+\lambda_{p}^{n}}{\left|r \frac{\partial \boldsymbol{x}_{p-1}^{n}}{\partial s}+\lambda_{p}^{n}\right|} .
$$

On the other hand, problem $[19)_{2}$ solution is obtained solving

$$
\begin{gathered}
\int_{0}^{L} E I \frac{\partial^{2} \boldsymbol{x}_{p}^{n}}{\partial s^{2}} \cdot \frac{\partial^{2} \boldsymbol{\xi}}{\partial s^{2}} d s+r \int_{0}^{L} \frac{\partial \boldsymbol{x}_{p}^{n}}{\partial s} \cdot \frac{\partial \boldsymbol{\xi}}{\partial s} d s+\frac{2}{\delta t^{2}} \int_{0}^{L} m \boldsymbol{x}_{p}^{n} \cdot \boldsymbol{\xi} d s= \\
\int_{0}^{L} \boldsymbol{f}^{n} \cdot \boldsymbol{\xi} d s+\frac{1}{\delta t^{2}} \int_{0}^{L} m\left(5 \boldsymbol{x}^{n-1}-4 \boldsymbol{x}^{n-2}+\boldsymbol{x}^{n-3}\right) \cdot \boldsymbol{\xi} d s+\int_{0}^{L}\left(r \mathbf{q}_{p}^{n}-\lambda_{p}^{n}\right) \cdot \frac{\partial \boldsymbol{\xi}}{\partial s} d s \quad \forall \boldsymbol{\xi} \in K .
\end{gathered}
$$

Remark 2 The penalization parameter $r$ plays a fundamental role in the convergence of the algorithm. If $r$ is smaller than a certain value $r_{c}$, (19) does not converge. If $r$ is too large, (19) has a slow convergence, can lead to local solutions of the minimization problem and can give rise to non-physical rigidity. We refer to [2] for further discussions on this issue.

\subsubsection{Space discretization}

The discretization of (19) $)_{2}$ is done using a third order Hermite finite element method in order to obtain an internal approximation of $H^{2}(0, L)$. We mesh the segment $[0, L]$ with $N_{\Sigma}-1$ elements $\left[s_{i}, s_{i+1}\right]$, such that $s_{1}=0$ and $s_{N_{\Sigma}}=L$. We denote by $h_{i}$ the length of the interval $\left[s_{i}, s_{i+1}\right]$ and we introduce the basis functions space

$$
Z_{h}=\left\{v_{h} \in C^{1}([0, L]):\left.v_{h}\right|_{\left[s_{i}, s_{i+1}\right]} \in \mathbb{P}_{3}, 1 \leq i \leq N_{\Sigma}-1\right\},
$$

and the set of allowed displacements

$$
K_{h}=\left\{\boldsymbol{x}_{h} \in\left(Z_{h}\right)^{2}: \frac{\partial \boldsymbol{x}_{h}}{\partial s}=\mathbf{q}_{h},\left|\mathbf{q}_{h}\right|^{2}=1, \boldsymbol{x}_{h}(0)=0, \frac{\partial \boldsymbol{x}_{h}}{\partial s}(0)=0\right\} .
$$

\section{Fluid-structure coupling}

\subsection{General strategy}

A purpose of this work was to design a flexible strategy which (1) keeps distinct solvers for fluid and structure; (2) allows to mix several structure models; (3) allows to mix ALE and FD formulations; (4) manages contact, independently of the structure solvers. The global

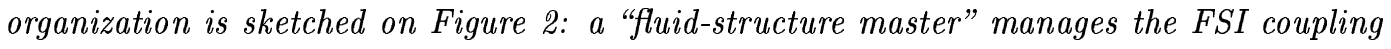




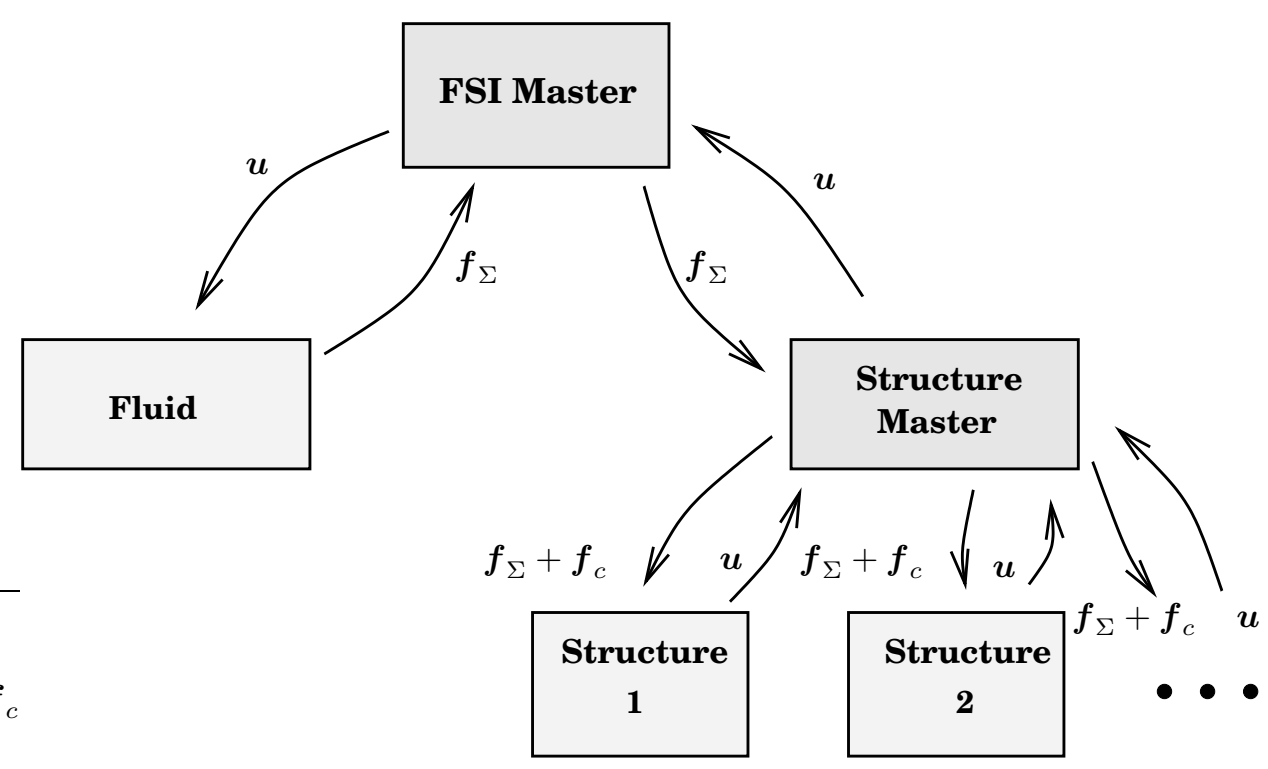

Figure 2: Global scheme of the partitioned strategy. The notation $\boldsymbol{u}$ stands for displacements and velocities, $f_{\Sigma}$ for the hydrodynamic forces, $f_{c}$ for the contact forces.

algorithm, while a "structure master" manages the structures and the contact. Whatever the coupling algorithm (loosely coupled, strongly coupled, etc.), whatever the fluid formulation (ALE, FD, or both), whatever the number and the kind of structures, in presence of contact or not, the only modification to perform in existing solvers are as limited as possible: for the fluid, it only consists in sending a load and receiving displacements/velocities whereas, for the structure it only consists in sending displacement/velocities and receiving a load.

\subsection{Hydrodynamic force computation}

For the numerical stability, it is important to carefully compute the load exerted by the fluid on the structure. As proposed in [5], this computation can be performed in a way ensuring a global energy conservation at the discrete level.

As previously mentioned the structure discretization is based on third order Hermite polynomials. We denote by $\boldsymbol{u}_{\Sigma^{n+1}, h}^{n+1}$ the discrete structure velocity, and by $U_{\Sigma} \in\left(\mathbb{R}^{2 N_{\Sigma}}\right)^{2}$ the vector of the components of $\boldsymbol{u}_{\Sigma^{n+1}, h}^{n+1}$ with respect to the basis $\left(\phi_{1}^{S}, \ldots, \phi_{N_{\Sigma}}^{S}, \phi_{N_{\Sigma}+1}^{S}, \ldots, \phi_{2 N_{\Sigma}}^{S}\right)$. We adopt the following convention: the first $N_{\Sigma}$ degrees of freedom correspond to the nodal values of the function, whereas the last $N_{\Sigma}$ degrees of freedom correspond to the values of its derivative. 
If the stress $\boldsymbol{f}_{\Sigma}^{n+1}=\left(f_{\Sigma}^{n+1,(k)}\right)_{k=1,2}$ defined in (2) was exactly known, the load transmitted to the discrete structure problem would have the form $F=\left(F_{1}^{(1)}, \ldots, F_{2 N_{\Sigma}}^{(1)}, F_{1}^{(2)}, \ldots, F_{2 N_{\Sigma}}^{(2)}\right) \in$ $\left(\mathbb{R}^{N_{\Sigma}}\right)^{2}$, with

$$
F_{i}^{(k)}=\int_{\Sigma^{n+1}} \phi_{i}^{S} f_{\Sigma}^{n+1,(k)}, \quad k=1,2, \text { and } i=1, \ldots, 2 N_{\Sigma},
$$

But $f_{\Sigma}^{n+1}$ is only known through a discrete form resulting from the discretization of the fluid problem. When an approximation of $\boldsymbol{f}_{\Sigma}^{n+1}$ is directly computed from the discrete solution $\left(\boldsymbol{u}_{h}, p_{h}\right)$, a spurious power may appear on the fluid structure interface. In some situations, we have noticed that this may deteriorate the stability of the coupling. To avoid this phenomenon, the computation of $F$ must be done in a way consistent with the discretization of the kinematic constraint (12) resulting from the choice of the Lagrange multiplier space (14). Let us make precise this statement.

For $k=1,2$, we denote by $u_{h}^{(k)}$ and $\lambda_{h}^{(k)}$ the $k^{\text {th }}$ components of the fluid velocity $\boldsymbol{u}_{h}$ and of the Lagrange multiplier $\lambda_{h}$, respectively. We denote by $U^{(k)} \in \mathbb{R}^{N_{F}}$ the vector of components of $u_{h}^{(k)}$ on the velocity finite element basis. Owing to the definition of the Lagrange multiplier space, and in view of (15), the algebraic counterpart of relation (12) reads

$$
K U^{(k)}=\mathcal{I} U_{\Sigma}^{(k)}, \quad k=1,2 .
$$

where $K \in \mathbb{R}^{N_{\Sigma} \times N_{F}}$ is the interpolation matrix of the structure nodes $\left(\mathbf{x}_{i}\right)_{i=1 . . N_{\Sigma}}$ on the fluid mesh, and $\mathcal{I} \in \mathbb{R}^{N_{\Sigma} \times 2 N_{\Sigma}}$ is the matrix whose entries are $\mathcal{I}_{i j}=\delta_{i j}$ if $j \leq N_{\Sigma}$ and 0 otherwise ( $\delta_{i j}$ being the Krönecker symbol).

We denote by $\Lambda^{(k)} \in \mathbb{R}^{N_{\Sigma}}$ the vector of the components of the Lagrange multiplier $\lambda_{h}^{(k)}$ on the basis $\left(\delta\left(\mathbf{x}_{i}^{n+1}\right)\right)_{i=1 . . N_{\Sigma}}$. The power exchanged at the interface "as seen by the fluid" is

$$
<\boldsymbol{\lambda}_{h}, \operatorname{Tr}_{\Sigma^{n+1}}\left(\boldsymbol{u}_{h}\right)>=\sum_{k=1}^{2}\left(\Lambda^{(k)}, K U^{(k)}\right)_{N_{\Sigma}},
$$

where $(\cdot, \cdot)_{N_{\Sigma}}$ denotes the euclidean scalar product in $\mathbb{R}^{N_{\Sigma}}$.

On the other hand, the power exchanged at the interface "as seen by the structure" is

$$
\int_{\Sigma^{n+1}} \boldsymbol{u}_{\Sigma^{n+1}, h}^{n+1} \cdot \boldsymbol{f}_{\Sigma}^{n+1} d l=\sum_{k=1}^{2}\left(F^{(k)}, U_{\Sigma}^{(k)}\right)_{2 N_{\Sigma}} .
$$

where $(\cdot, \cdot)_{2 N_{\Sigma}}$ denotes the euclidean scalar product in $\mathbb{R}^{2 N_{\Sigma}}$.

From (22) and (23), we deduce that the energy exchange between the fluid and the structure is well-balanced at the discrete level as soon as

$$
\left(F^{(k)}, U_{\Sigma}^{(k)}\right)_{2 N_{\Sigma}}=\left(\Lambda^{(k)}, K U^{(k)}\right)_{N_{\Sigma}}, \quad k=1,2 .
$$

Since (24) must be valid for all $\boldsymbol{u}_{\Sigma}$, relations (21) and (24) give the relation

$$
F=\mathcal{I}^{T} \Lambda \text {. }
$$


Owing to the definition of the matrix $\mathcal{I}$, we see in particular that the degrees of freedom on the derivative part of the Hermite basis are zero. This results from the fact that the kinematic constraint (12) discretized with the Lagrange multiplier space (14) "does not see" the derivative degree of freedom of the structure velocity.

\subsection{Contact force computation}

We assume that the contacts are frictionless and occur between the valve and a rigid straight wall. The situation is therefore rather academical and simple, the constraints being convex. The algorithm we consider is based on a duality argument which is well-established. The only purpose of this section is to show how contacts can be easily handled with the proposed fluidstructure algorithm, even when the structure solvers are not designed to manage contacts. Multi-body contacts, which are more involved (non convex constraints) but important for the applications, will be investigated in a forthcoming work. The algorithm presented here can be seen as a first step towards this goal.

We directly consider the problem in its discrete form. At each time step, we want to minimize a structure energy $\boldsymbol{X}=\left(\boldsymbol{x}_{1}, \ldots, \boldsymbol{x}_{N_{\Sigma}}\right) \rightarrow J(\boldsymbol{X})$ under the constraint $\boldsymbol{X} \in \mathcal{U}$, with

$$
\mathcal{U}=\left\{\boldsymbol{X}=\left(\boldsymbol{x}_{1}, \ldots, \boldsymbol{x}_{N_{\Sigma}}\right) \in\left(\mathbb{R}^{2}\right)^{N_{\Sigma}}, \varphi\left(\boldsymbol{x}_{i}\right) \leq 0, i=1, \ldots, N_{\Sigma}\right\}
$$

where $\varphi$ is a convex function and $\boldsymbol{x}_{i}$ denotes the position of the $i^{\text {th }}$ structure node. In this study, we only consider functions of the form:

$$
\varphi(\boldsymbol{x})=\boldsymbol{x} \cdot \boldsymbol{n}_{w}-d_{0},
$$

where $\boldsymbol{n}_{w}$ denotes the normal to the rigid straight wall and $d_{0}$ is a given constant coefficient. The energy $J$ is typically of the form (17). In particular, it includes the zero order terms resulting from the discretization of the acceleration and the work of the given hydrodynamic force $\boldsymbol{f}_{\Sigma}$.

Various methods to solve this problem - like penalization or relaxation with projection - may yield substantial changes of the structure solver. Since we would like to keep as low as possible the modifications in existing solvers, we adopt a dual approach which consists in maximizing the dual energy

$$
G(\boldsymbol{\mu})=\inf _{\boldsymbol{X} \in\left(\mathbb{R}^{2}\right)^{N_{\Sigma}}}\left(J(\boldsymbol{X})+\sum_{i=1}^{N_{\Sigma}} \mu_{i} \varphi\left(\boldsymbol{x}_{i}\right)\right),
$$

for $\boldsymbol{\mu}=\left(\mu_{1}, \ldots, \mu_{N_{\Sigma}}\right) \in \mathbb{R}^{N_{\Sigma}}, \boldsymbol{X}=\left(\boldsymbol{x}_{1}, \ldots, \boldsymbol{x}_{N_{\Sigma}}\right) \in \mathbb{R}^{2 N_{\Sigma}}$, under the constraints

$$
\mu_{i} \geq 0, i=1, \ldots, N_{\Sigma} \text {. }
$$

From the mechanical viewpoint, the Lagrange multipliers $\mu_{i}$ represent the contact forces. In a gradient method with projection, these constraints are very easy to implement, whereas the original one, namely $\boldsymbol{X} \in \mathcal{U}$, may be complicated. This is the standard motivation of the 
dual approach. In our specific framework, this method has another advantage: the structure solver exchanges the same kind of information with the master as for the coupling with the fluid (it receives loads, it sends displacements). The contact treatment can therefore be easily included as an inner-loop in the global algorithm. We can sum up the contact algorithm as follows:

(i) Given an hydrodynamic force $\boldsymbol{f}_{\Sigma}$

(ii) Solve the structure problem with the hydrodynamic force and the contact force:

$$
\left(J^{\prime}\left(\boldsymbol{X}_{\boldsymbol{\mu}^{k}}\right), \boldsymbol{\xi}\right)=-\sum_{i=1}^{N_{\Sigma}} \mu_{i}^{k} \boldsymbol{\xi} \cdot \boldsymbol{n}_{w}
$$

(iii) Update the Lagrange multiplier:

$$
\mu_{i}^{k+1}=\left(\mu_{i}^{k}+\alpha\left(\nabla G\left(\boldsymbol{\mu}^{k}\right)\right)_{i}\right)_{+}, \quad i=1, \ldots, N_{\Sigma},
$$

where $\alpha$ denotes a positive given parameter, $(x)_{+}=\max (x, 0)$ and

$$
(\nabla G(\mu))_{i}=\varphi\left(\boldsymbol{x}_{\boldsymbol{\mu}^{k}, \boldsymbol{i}}\right) .
$$

For example, assume that we start from a state without contact $\left(\mu_{i}^{0}=0\right.$ for $\left.i=1, \ldots, N_{\Sigma}\right)$. If no contact occurs, then $\varphi\left(\boldsymbol{x}_{\boldsymbol{\mu}^{0}, i}\right)<0$ thus $\mu_{i}^{0}+\alpha \varphi\left(\boldsymbol{x}_{\boldsymbol{\mu}^{0}, i}\right)<0$ and step (ii) gives $\mu_{i}^{1}=0$. If a contact occurs, then for some $i$, we have $\varphi\left(\boldsymbol{x}_{\mu^{0}, i}\right)>0$ and step (ii) gives a positive contact force.

\subsection{Global algorithm}

We sum up on Figure 3 the general algorithm. To speed up the convergence of the fixed point iterations, we used an Aitken acceleration technique. We refer to [18] for the details. In our experience, this method is efficient enough when the structure is an immersed valve. But as soon as the elasticity of the wall is also taken into account (as in Section 5.4) more efficient methods have to be designed.

\section{NUMERICAL RESULTS}

\subsection{Comparison with ALE formulations}

In order to ascertain the accuracy of the presented Fictitious Domain method applied to valves immersed in a Navier-Stokes fluid, we propose a comparison with a simulation based on an Arbitrary Lagrangian Eulerian formulation (ALE). The model chosen for the valve is the inextensible $1 D$ structure described above, clamped on a rigid wall (see Fig. 1). While the 


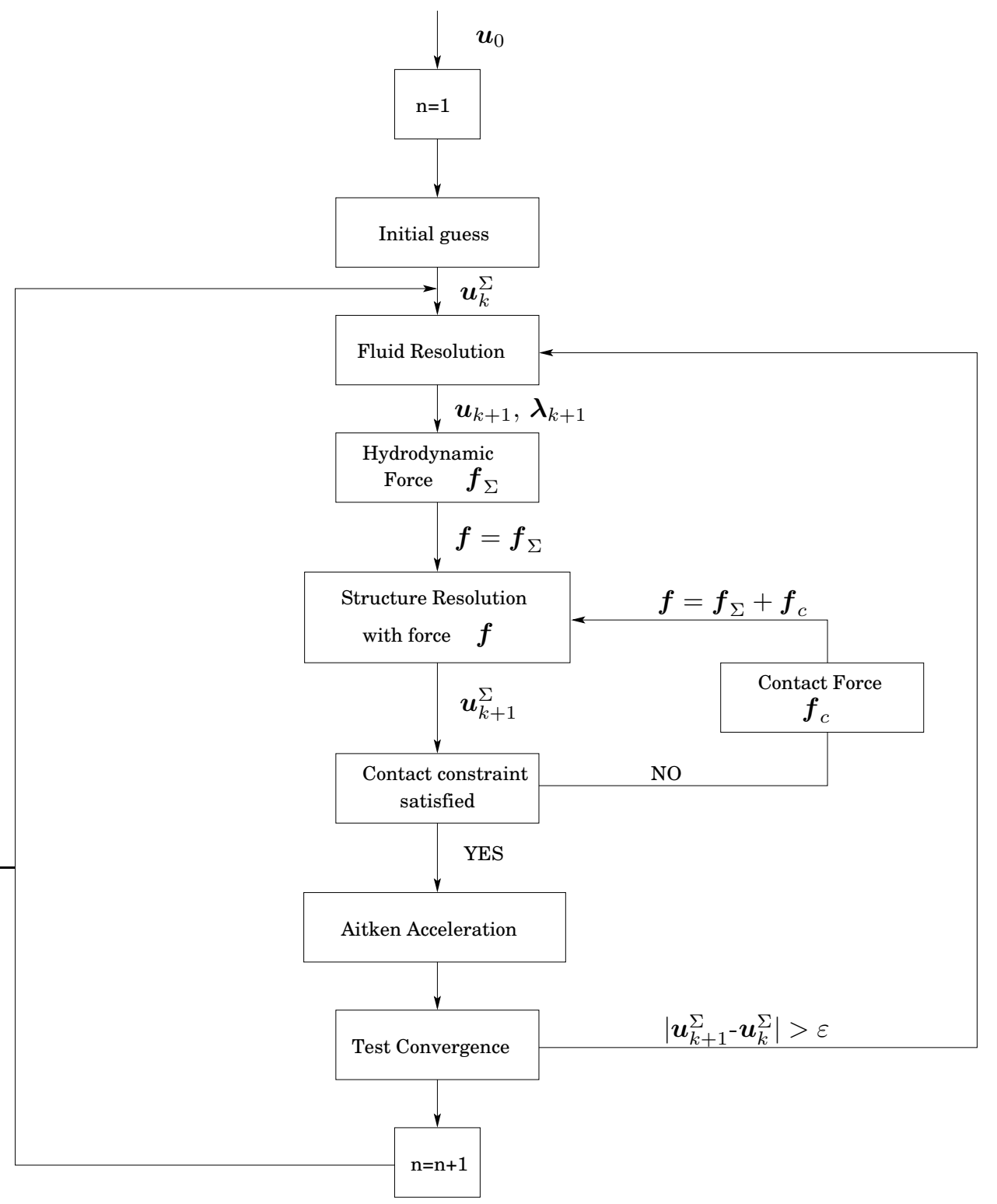

Figure 3: General algorithm. 
flow causes the immersed structure to bend and to have different configurations throughout the cycle, the flow is also greatly affected by the movement of the valve. A pressure difference is applied between the inlet $\left(\Gamma_{\text {in }}\right)$ and the outlet $\left(\Gamma_{\text {out }}\right)$. The pressure $p_{\text {out }}$ is zero. The pressure $p_{i n}$ is a continuous function of time $t$, periodic with a period 0.8, and defined by:

$$
p_{\text {in }}(t)= \begin{cases}A & \text { if } 0.0125 \leq t \leq 0.3875 \\ -A & \text { if } 0.4125 \leq t \leq 0.7875 \\ \text { affine } & \text { if } t \in[0,0.0125] \text { or }[0.3875,0.4125] \text { or }[0.7875,0.8]\end{cases}
$$



Figure 4: Comparison between the iso-values of the velocity. The behavior of both methods, FD (up) and ALE (down) is almost identical.

We consider a stiff valve (Fig. (4). The displacements are moderate enough to avoid remeshing when the ALE method is used. The data considered are: $\rho=1.0 \mathrm{~g} \mathrm{~cm}^{-3}, \eta=$ 0.1 poise, $E I=0.7 \times 10^{-2} \mathrm{~g} \mathrm{~cm}^{3} \mathrm{~s}^{-2}$ (flexural stiffness), $m=2.5 \mathrm{~g}$ and the pressure function (26) with amplitude $A=40 \mathrm{~g} \mathrm{~cm}^{-1} \mathrm{~s}^{-2}$ is imposed at the inlet. The length of the valve is $L=0.45 \mathrm{~cm}$, the tube is $6 \mathrm{~cm}$ long and $1 \mathrm{~cm}$ high. FD and $A L E$ methods are compared using the same meshes at the initial time step. The ALE reference computation was performed 

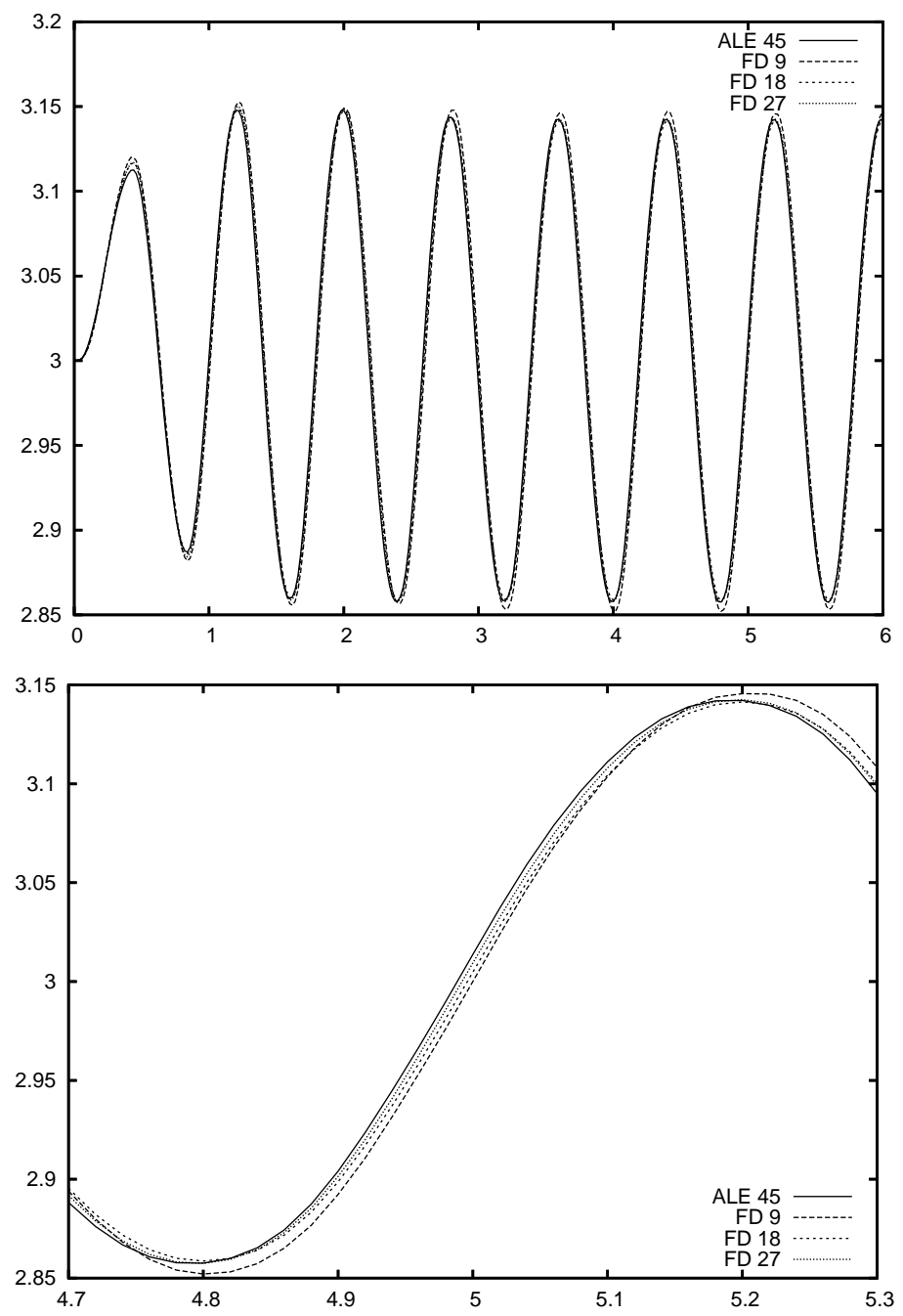

Figure 5: Comparison of the movement of the apex for ALE and FD methods with different space steps. The bottom graphic shows a zoom where the difference between 9 space steps and the remainder becomes more visible.

with 45 space discretization steps on the valve $\left(h_{s}=L / 45=0.01 \mathrm{~cm}\right)$. The time step is $5 \times 10^{-3} \mathrm{~s}$.

We compare the displacement of the valve's apex and the load on the structure obtained by both methods. For the displacement, the results can be seen on Figure 5 and on Table 1 


\begin{tabular}{|c|c|c|}
\hline$h_{s}$ & Method & error \\
\hline \hline$L / 9$ & ALE & 0.00441 \\
& FD & 0.00502 \\
\hline$L / 18$ & ALE & 0.00345 \\
& FD & 0.00454 \\
\hline$L / 27$ & ALE & 0.00216 \\
& FD & 0.00333 \\
\hline
\end{tabular}

Table 1: The relative error of the displacement is evaluated on the $L^{\infty}\left(0, T ; L^{\infty}(\Sigma)\right)$ norm. The reference solution is obtained using the ALE with $h_{s}=\frac{L}{45}$. The relative errors are then shown for different space steps $h_{s}$.

For the loads, using the $L^{\infty}$ norm, we found a $6 \%$ error between the FD simulation with $h_{s}=L / 27$ and the reference ALE simulation. A qualitative comparison of the velocity profiles is also presented on Figure 4.

\subsection{Sensitivity to discretization steps}

For the fictitious domain method, we observed a significant relative error on the displacement of the valve with 9 or 18 space discretization steps. For the the remaining space steps (27, 36, 45 and 54) the differences, even if present, were minor.
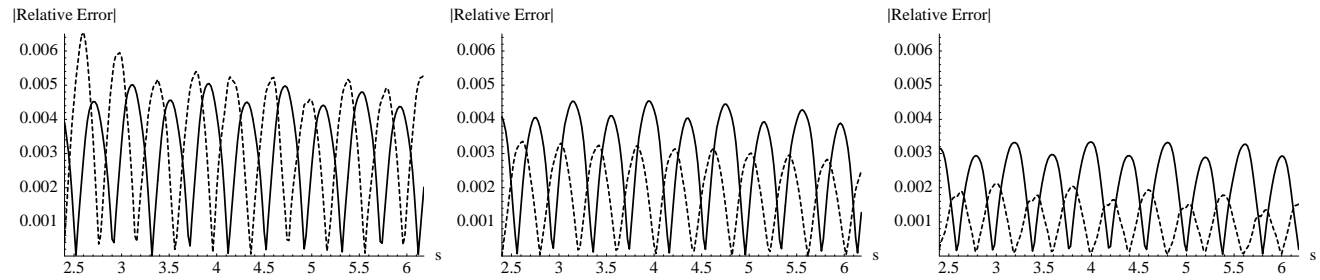

Figure 6: Relative error in time of the position of the valve apex. The error is computed with respect to a reference solution obtained with the ALE formulation with 45 space steps on the structure. From the left-hand side to the right-hand side, we consider 9,18 and 27 space steps. The continuous line represents ALE and the dashed represents FD.

\subsection{A simulation with contact}

We now propose two test cases with large displacements as well as contact on an artificial wall. The contact problem without friction is solved with the dual approach method described 
in Section 4.3. In the first experiment (Fig. (7) the $1.13 \mathrm{~cm}$ valve is embedded at 60 degrees. When it closes, it completely impedes the fluid flow. The valve stiffness is $E I=1.0 \mathrm{~g} \mathrm{~cm}^{3} \mathrm{~s}^{-2}$ and the pressure (26) has an amplitude $A=200 \mathrm{~g} \mathrm{~cm}^{-1} \mathrm{~s}^{-2}$.

In the second example (Fig. [8) the valve is less stiff. It has very large displacements and is allowed to touch the top artificial wall. The length of the tube is $6 \mathrm{~cm}$, its height is $1 \mathrm{~cm}$. The valve length is $1.1 \mathrm{~cm}$, its flexural stiffness is $E I=2.5 \times 10^{-2} \mathrm{~g} \mathrm{~cm}^{3} \mathrm{~s}^{-2}$ and the pressure (26) has an amplitude $A=140 \mathrm{~g} \mathrm{~cm}^{-1} \mathrm{~s}^{-2}$.

\subsection{Mix ALE and FD}

In this last test we show that the proposed FD method for the valve can be conveniently mixed with an ALE formulation to handle elastic walls (Fig. (9). The domain is now time dependent $\Omega(t)$; the fluid is therefore solved in the ALE formulation (see [10] for example). One side of $\Omega(t)$ is occupied by an elastic wall whose behavior is governed by a generalized string equation:

$$
\rho_{w} h \frac{\partial^{2} d}{\partial t^{2}}-k G h \frac{\partial^{2} d}{\partial z^{2}}+\frac{E h}{1-\nu^{2}} \frac{d}{R_{0}^{2}}-\gamma \frac{\partial^{3} d}{\partial z^{2} \partial t}=f_{\Sigma},
$$

where $d$ denotes the vertical displacement of the wall, $h$ is the wall thickness, $k$ is the socalled Timoshenko shear correction factor, $G=E /(2+2 \nu)$ the shear modulus, E the Young modulus, $\nu$ the Poisson ratio, $\rho_{w}$ the wall density and $\gamma$ a viscoelastic parameter. The following parameters are used: $E=0.75 \cdot 10^{6}$ dynes $/ \mathrm{cm}^{2}, \nu=0.5, \rho_{S}=1.1 \mathrm{~g} / \mathrm{cm}^{3}, h=$ $0.1 \mathrm{~cm}, R_{0}=0.5 \mathrm{~cm}, k=1$. The valve is modelled as in the previous test cases. Its length is $0.49 \mathrm{~cm}$ and its flexural stiffness is $E I=0.5 \mathrm{~g} \mathrm{~cm}^{3} \mathrm{~s}^{-2}$. The inlet pressure $p_{\text {in }}$ is defined by $p_{\text {in }}(t)=2000$ if $0 \leq t<0.01, p_{\text {in }}(t)=-2000$ if $0.06 \leq t<0.08$, and $p_{\text {in }}(t)=0$ otherwise.

The compliance of the wall is responsible for the propagation of a pressure wave which interacts with the valve as shown on Figure [9 Now the "Structure Master" (see Fig. Q) manages two structure - the valve and the wall - but the coupling algorithm is exactly the same as before (Fig. (3). This demonstrates the flexibility of the proposed approach. As mentioned in Section 4.4, the presence of the compliant wall increases significantly the number of fixed point iterations. More efficient coupling algorithms should be used in that case. This will be done in a future work, based on the ideas proposed in [7, 8].

\section{CONCLUSIONS}

In this paper we have presented a Fictitious Domain approach and a partitioned strategy to solve the coupling between an immersed thin structure and an incompressible fluid. Numerical tests have demonstrated a good agreement with ALE simulations in the case of moderate displacements and a good robustness in the case of very large displacements. Furthermore, the present approach also proved well-suited to be used simultaneously with an ALE formulation in presence of elastic walls. Simple contact constraints can be conveniently introduced 
in the partionned algorithm. Valve/valve or valve/moving wall contacts will be investigated in a future work.

\section{Acknowledgments}

This work has been partially supported by the Research Training Network «Mathematical Modelling of the Cardiovascular System» (HaeMOdel), contract HPRN-CT-2002-00270 of the European Community.

\section{References}

[1] F.P.T. Baaijens. A fictitious domain/mortar element method for fluid-structure interaction. International Journal for Numerical Methods in Fluids, 35:743-761, 2001.

[2] J.-F. Bourgat, J.M. Dumay, and R. Glowinski. Large displacement calculations of flexible pipelines by finite element and nonlinear programming methods. Journal on Scientific Computing, 1:34-81, 1980.

[3] J. De Hart, F.P.T. Baaijens, G.W.M. Peters, and P.J.G. Schreurs. A computational fluid-structure interaction analysis of a fiber-reinforced stentless aortic valve. Journal of Biomechanics, 36:699-712, 2003.

[4] J. De Hart, G.W.M. Peters, P.J.G. Schreurs, and F.P.T. Baaijens. A threedimensional computational analysis of fluid-structure interaction in the aortic valve. Journal of Biomechanics, 36:103-112, 2003.

[5] C. Farhat, M. Lesoinne, and P. Le Tallec. Load and motion transfer algorithms for fluid/structure interaction problems with non-matching discrete interfaces: Momentum and energy conservation, optimal discretization and application to aeroelasticity. Comput. Methods Appl. Mech. Engrg., 157:95-114, 1998.

[6] M. Fernández, A. Moura, and C. Vergara. Defective boundary conditions applied to multiscale analysis of blood flow. ESAIM Proceedings, 14:89-99, 2005.

[7] M.A. Fernández, J.-F. Gerbeau, and C. Grandmont. A projection algorithm for fluidstructure interaction problems with strong added-mass effect. C. R. Acad. Sci. Paris, Math., 342:279-284, 2006.

[8] M.A. Fernández, J.-F. Gerbeau, and C. Grandmont. A projection semi-implicit scheme for the coupling of an elastic structure with an incompressible fluid. Int. J. Num. Meth. Engng. in press, 2006. 
[9] L. Formaggia, J.-F. Gerbeau, F. Nobile, and A. Quarteroni. Numerical treatment of defective boundary conditions for the navier-stokes equations. SIAM J. Numerical Analysis, 40(1):376-401, 2002.

[10] J.-F. Gerbeau and M. Vidrascu. A quasi-newton algorithm based on a reduced model for fluid-structure interactions problems in blood flows. Math. Model. Num. Anal., $37(4): 631-648,2003$.

[11] R. Glowinski, T.-W. Pan, T.I. Hesla, and D.D. Joseph. A distributed lagrange multiplier/fictitious domain method for particulate flows. International Journal of Multiphase Flow, 25:755-794, 1999.

[12] R. Glowinski, T.-W. Pan, T.I. Hesla, D.D. Joseph, and J. Périaux. A fictitious domain approach for the direct simulation of incompressible fluid flow past moving rigid bodies: Application to particulate flow. Journal of Computational Physics, 169:363-426, 2001.

[13] R. Glowinski, T.-W. Pan, T.I. Hesla, and J. Périaux. A fictitious domain method for dirichlet problem and applications. Computational Methods in Applied Mechanics and Engineering, 111:283-303, 1994.

[14] Z. Jianhai, C. Dapeng, and Z. Shengquan. ALE finite element analysis of the opening and closing process of the artificial mechanical valve. Applied Mathematics and Mechanics, 17(5):403-412, 1996.

[15] P. Le Tallec and J. Mouro. Fluid structure interaction with large structural displacements. Comput. Meth. Appl. Mech. Engrg., 190:3039-3067, 2001.

[16] C.F. Loeffler and W.J. Mansur. Analysis of time integration schemes for boundary element applications to transient wave propagation problems. In Southampton U.K., editor, BETECH'87. Computational Mechanics Publications, 1996.

[17] R. Loon, P.D. Anderson, J. De Hart, and F.P.T. Baaijens. A combined fictitious domain/adaptative meshing method for fluid-structure interaction in heart valves. International Journal for Numerical Methods in Fluids, 46:533-544, 2004.

[18] D. P. Mok, W. A. Wall, and E. Ramm. Accelerated iterative substructuring schemes for instationary fluid-structure interaction. In K.J. Bathe, editor, Computational Fluid and Solid Mechanics, pages 1325-1328. Elsevier, 2001.

[19] C. Peskin. The immersed boundary method. Acta Numerica - Cambridge University Press, pages 1-39, 2002.

[20] V. C. Sripathi, R. Krishna Kumar, and K.R. Balakrishnan. Further insights into normal aortic valve function: Role of a compliant aortic root on leaflet opening and valve orifice area. Ann. thorac. surg., 77(3):844-851, 2004.

[21] Z. Yu. A DLM/FD method for fluid/flexible-body interactions. J. Comput. Phys., 207(1):1-27, 2005. 


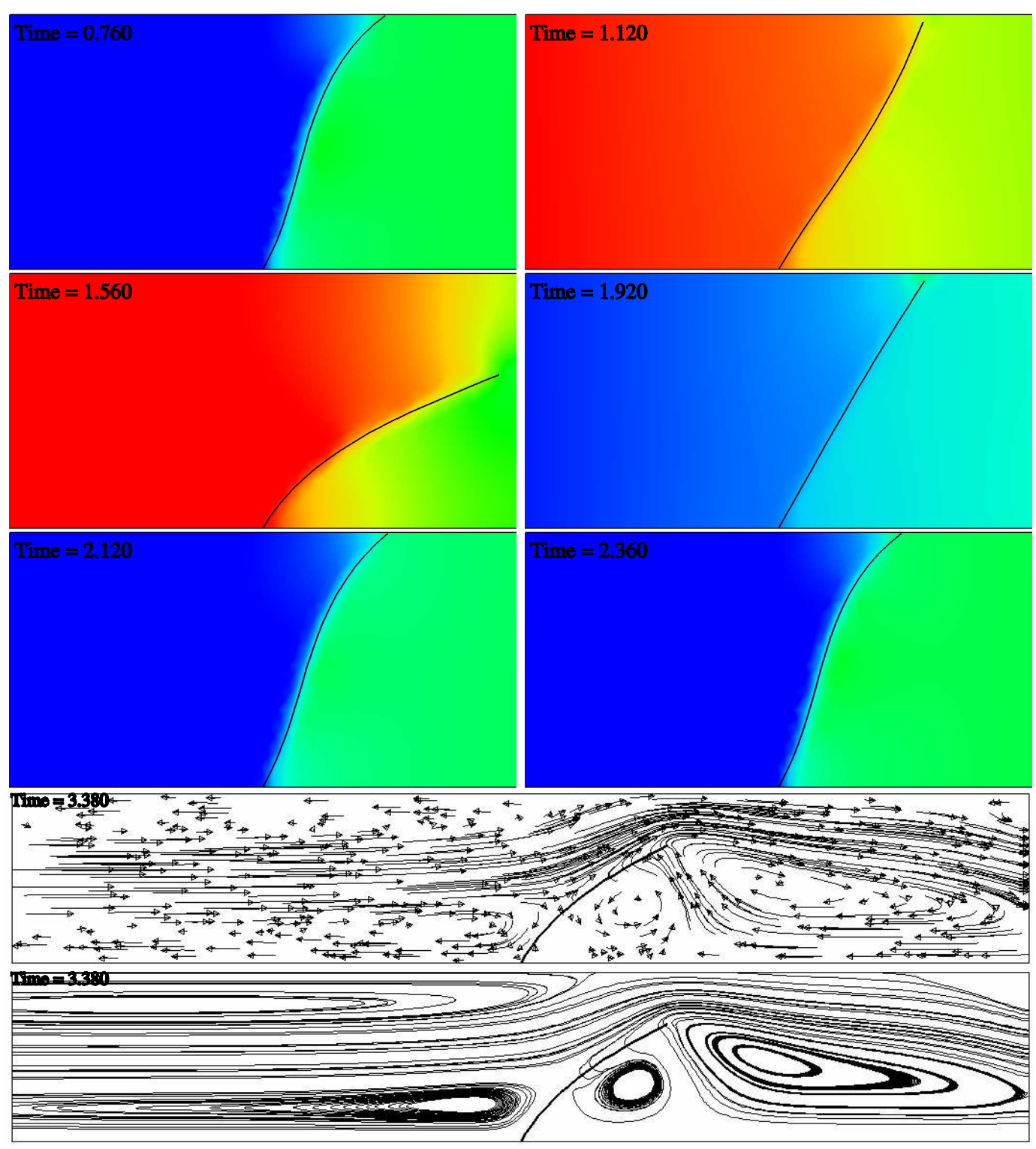

Figure 7: Snapshots of the contact problem when it impedes the fluid flow. The six first figures show, at six different times, the pressure around the valve. The last picture shows the velocity and the streamlines at time $3.38 s$ when the valve is starting to close. The colors reflect the difference of the pressures between the left and the right-hand side of the valve. 


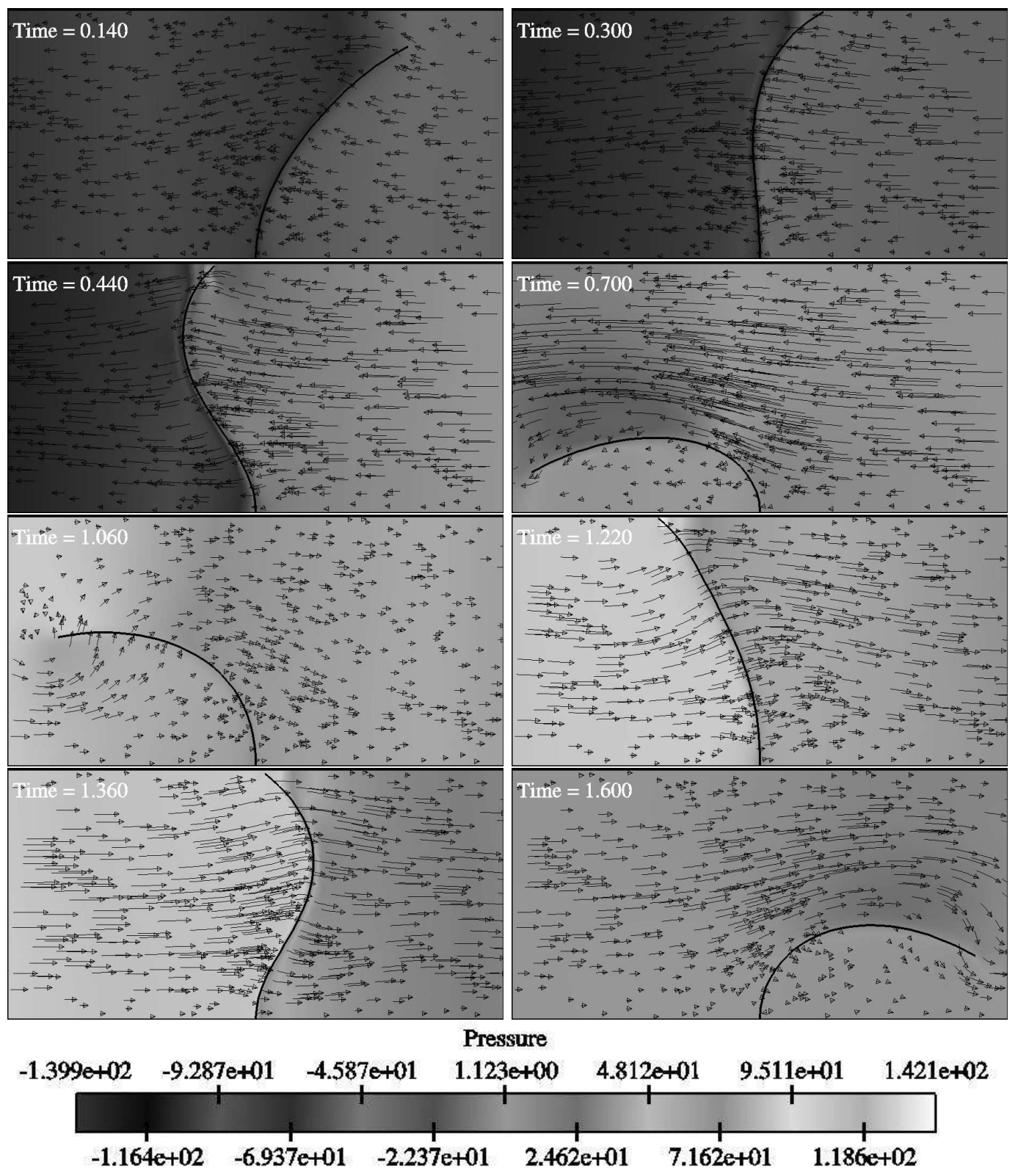

Figure 8: Snapshots of the contact problem with a very compliant valve. The arrows represent the velocity and the size is proportional to its intensity. The domain shown here of $2 \mathrm{~cm} \times 1 \mathrm{~cm}$ is a sample of a $6 \mathrm{~cm} \times 1 \mathrm{~cm}$ tube. 

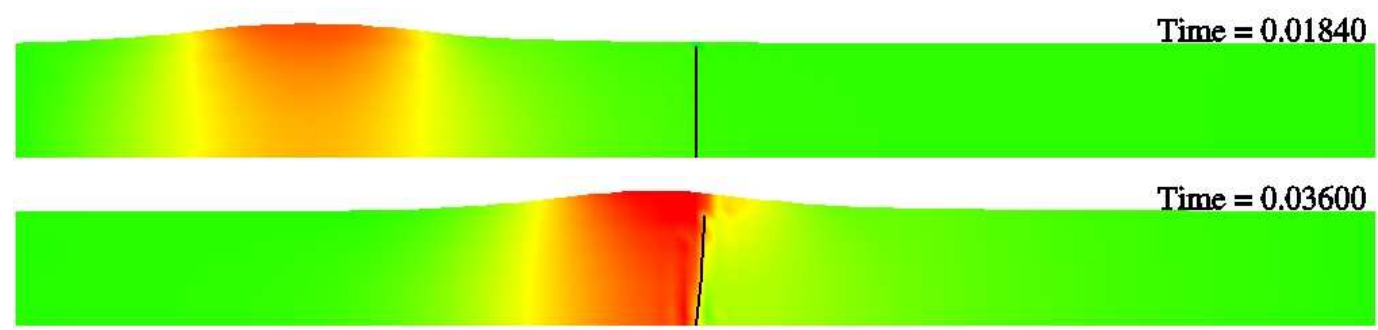

Time $=0.06000$

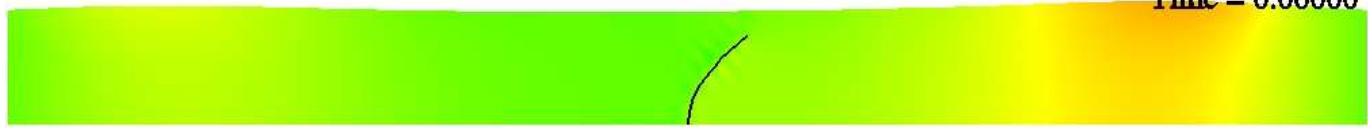

Time $=0.07680$

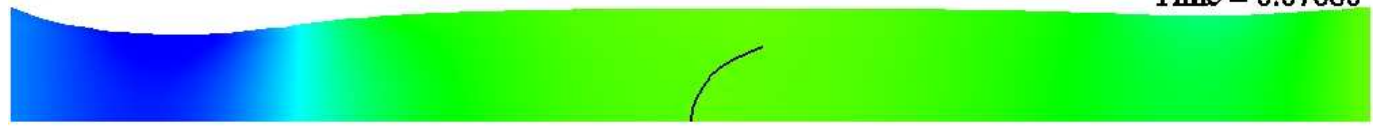

Time $=0.10640$

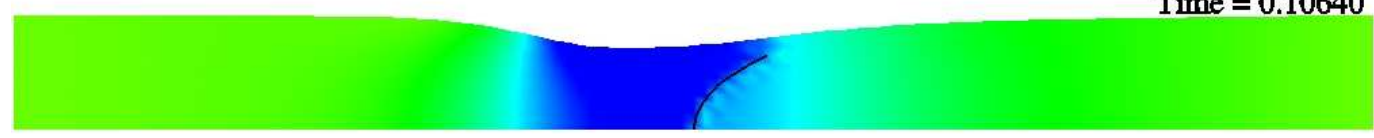

Time $=0.12800$
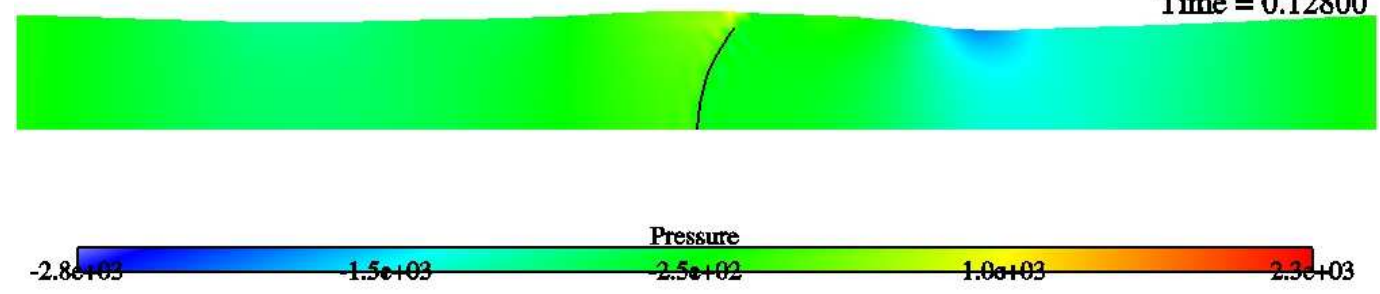

Figure 9: Interaction of a pressure wave with a valve. The simulation is obtained by mixing ALE (for the elastic wall) and FD (for the valve). 




Unité de recherche INRIA Rocquencourt

Domaine de Voluceau - Rocquencourt - BP 105 - 78153 Le Chesnay Cedex (France)

Unité de recherche INRIA Futurs : Parc Club Orsay Université - ZAC des Vignes

4, rue Jacques Monod - 91893 ORSAY Cedex (France)

Unité de recherche INRIA Lorraine : LORIA, Technopôle de Nancy-Brabois - Campus scientifique

615, rue du Jardin Botanique - BP 101 - 54602 Villers-lès-Nancy Cedex (France)

Unité de recherche INRIA Rennes : IRISA, Campus universitaire de Beaulieu - 35042 Rennes Cedex (France)

Unité de recherche INRIA Rhône-Alpes : 655, avenue de l'Europe - 38334 Montbonnot Saint-Ismier (France)

Unité de recherche INRIA Sophia Antipolis : 2004, route des Lucioles - BP 93 - 06902 Sophia Antipolis Cedex (France)

INRIA - Domaine de Voluceau - Rocquencourt, BP 105 - 78153 Le Chesnay Cedex (France)

http://www.inria.fr

ISSN 0249-6399 